\title{
The curse of the cure
}

\author{
Vincent T DeVita, Jr
}

We are in some ways victims of our own success in the management of childhood leukemia and advanced Hodgkin's disease (HD). Leukemia was the first childhood malignancy to be cured by chemotherapy, and HD was the first tumor of a major organ system in adults, in its advanced stages, to be cured by chemotherapy. In both instances, as the majority of patients respond to chemotherapy, investigators are faced with the challenge of reducing the toxicity of treatment while still offering patients the maximum chance of cure. Both malignancies were also highly curable long before we knew any significant amount about their biology. Now we do. And the information offers us attractive molecular targets that may provide effective, and far less toxic, treatments than are available currently.

In this issue of the journal, Re et al. review some of the new enticing targets in HD that have been uncovered as we have finally identified the cell of origin. But they also raise some disturbing issues. First, we don't quite know where to test new therapies. The highly resistant malignant cell in any cancer, in vivo, is no better as a model than cells grown in vitro and is as likely to mislead us as to enlighten us. Patients who have not been previously treated are, therefore, considered the best experimental model for testing new approaches, but can we include new treatments early in any curable cancer without facing an ethical dilemma?

Second, our success in translating empiric treatments into practice, by training legions of oncologists, especially in the US, has proven a handicap to testing and adopting new methods. Many practicing oncologists are conservative. They tend to use tried and tested treatments in newly diagnosed patients, rather than enter
Future patients

deserve an

approach to

improving

the quality of

their lives as

novel as the

targets we now

know exist, to

overcome the

curse of the

cure

VT DeVita, Jr is the Editor-in-Chief of Nature Clinical Practice Oncology.

\section{Competing interests}

The author declared he has no competing interests.

www.nature.com/clinicalpractice doi:10.1038/ncponc0054 them into clinical studies. The fact that a significant fraction of patients with these diseases may be cured with the existing regimens has frozen their management in time.

Even newer versions of older approaches are resisted despite superior results. For example, as Re et al. point out, the dose-escalated BEACOPP program has proven effective in over $90 \%$ of patients with advanced HD, but it is not used that much in the US as it is more toxic. The math indicates, however, that the overall toxicity is not severe enough to offset its increased effectiveness.

We faced a similar situation in 1964, when we were developing the first curative treatment for advanced HD. Then, as now, we had difficulty recruiting previously untreated patients for clinical trials, as almost all the hematologists were using chlorambucil, a non-curative but easy-to-use treatment, first. We were eventually successful, and the more toxic but curative MOPP program emerged. As subsequent experience showed, we would have missed its effect had it been tested solely in heavilypretreated patients with refractory disease.

This is less of a problem in Europe, where the very successful German Hodgkin's Lymphoma Study Group dominates the study of this disease, albeit by parsing out large numbers of previously untreated patients democratically across specialties.

Thus, we need both new ways to test novel treatments in treatable malignancies like HD and a willingness to consider change by participating in clinical trials driven only by a hypothesis. We owe future patients an approach to improving the quality of their lives as novel as the new targets we now know exist, to overcome the curse of the cure. 